\title{
Polyurethane and Polyurethane Nanocomposites: Recent Contributions to Medicine ${ }^{\dagger}$
}

\author{
Dorel Feldman $1, *$ (i) \\ 1 Concordia University, Faculty of Engineering and Computer Science, 1455 De Maisonneuve Blvd West EV 6-403, \\ Montreal, Quebec, Canada, H3G 1M8 \\ * Correspondence: feldman@bcee.concordia.ca; \\ $\dagger \quad$ In memoriam Edith \& Tony
}

Scopus Author ID 7402702864

Received: 19.05.2020; Revised: 12.07.2020; Accepted: 15.07.2020; Published: 19.07.2020

\begin{abstract}
Polyurethane (PU) is a synthetic polymer obtained by polycondensation process of isocyanates and polyols, and for some cross-linked products with a third component as an extender. It is one of the most versatile macromolecular compounds, and it contains soft and hard segments. Beside (PU)'s physicomechanical and chemical properties, which make them suitable to be used in many engineering areas, they are biocompatible, biodegradable, bioabsorbant, and bioinert, characteristics which recommend them to be used in different medical areas. For instance, PUs and, more recently, their nanocomposites are used for wound dressing, antibacterials, tissue engineering, scaffolds, drug delivery, and even medical devices.
\end{abstract}

Keywords: Polyurethane; polyurethane nanocomposites; antibacterials; tissue engineering; drug carriers.

Abbreviations: AFM-Atomic force microscopy; APTT-Activated prothrombin time; APU-Smart PU foam (auxetic) PU; CA.-Cynnamaldehyde; CAP-Compound action potential; CCK-Cell counting kit; CS.-Chitosan; DEA-Diethanolamine; DH-Se-Di-(1-hydroxylundecyl)-selenide; DMA-Dinamo mechanical analysis; DMG-Dimethylol propionamide with guanidine; DOX-Doxorubicin (drug); EDXRF-Energy dispersive X-ray fluorescence; FTIR-Fourier transform infrared analysis; FDA-Food and Drug Administration; 5FU-5-Fluorouracil; G-Graphene; GO-Graphene oxide; LDI-Lisine-ethyl ester diisocyanate; MMA-Methylmethacrylate; MOF-Metal-organic-frameworks; MPU-Multiblock polyurethane; MTT-Cell proliferation-assay kit; nAg-Silver nanoparticle; oMMT-organic Montmorollonite; PAA-Lap-Poly(acrylic acid)-laponite; PAHU-Poly(amide hydroxyl urethane); PCLPoly(E-caprolactone); PDA-Polydopamine; PEBA-Poly(ether-block-amide); PEG-Poly(ethylene glycol); PGE-Poly(glycerol ester) PPG-Poly(propylene glycol); TPT-Thromboplastine timePUPolyurethane; QAP-Quaternary ammonium salt polymer; QAS-Quaternary ammonium salt diol; SEMScanning electron microscopy; SMP-Shape memory polymer; TGA-Thermogravimetric analysis; TPUThermoplastic polyurethane; WPU-Waterborne polyurethane; WPUD-Waterborne polyurethane dispersion; WVTR-Water vapor transmission rate.

(C) 2020 by the authors. This article is an open-access article distributed under the terms and conditions of the Creative Commons Attribution (CC BY) license (https://creativecommons.org/licenses/by/4.0/).

\section{Introduction}

PU is one of the most versatile macromolecular compounds which stands out with a wide range of tuneable characteristics such as chemical stability, flexibility, abrasion, scratch resistance, toughness, and biodegradability. It can contain various functional groups such as ether, ester, amide, urea, biuret, allophane, uretidione, carbodiimide, isocyanurate, along with urethane which opens possibilities to react with many other chemicals. 
PUs are synthesized by polycondensation in which the polymer chain increases as the reaction progresses. This process can lead to the production of linear, slightly branched, or hyperbranched macromolecules such as in thermoplastic polymers, or in the presence of an extender, they can form a cross-linked network leading to thermosetting.

PU contains in its macromolecule soft and hard segments; the former is made by the isocyanate - polyol reaction and the latter by the isocyanate-chain extender reaction.

Besides the engineering field, due to their properties such as controllable structure, biocompatibility, biodegradability, bioabsorbability, bioinertness. (PU)s and their nanocomposites can be used in medicine for wound dressing, antibacterials, tissue engineering, scaffolds, drug delivery, and biomedical devices [1-3].

\section{Polyurethane}

\subsection{Antibacterials.}

In order to produce hygiene requirements, many studies are done on antibacterial products based on PU.

Cationic waterborne PU(CWPU) was synthesized from waste frying oil and used as antibacterial film coatings. The obtained CWPU films provided excellent antibacterial activity with efficiency increasing with the increasing amount of bis (2-hydroxymethyl) ammonium chloride. Under similar conditions, antibacterial activity against Staphylococcus aureus was more rapid than that against Escherichia coli. [4].

By using the solvent-casting/particulate leaching technique combined with thermally induced phase separation, antibacterial microporous PU scaffolds, as thin layers were obtained. These scaffolds were modified with cinnamaldehyde (CA) to establish the most suitable antibacterial effect. CA-modified microporous PU thin-layer scaffold was found to be effective at a $3.5 \%$ concentration of $\mathrm{CA}[5]$.

A polyol based on itaconic acid and 1,6 hexanediol were used for waterborne PU dispersion (WPUDs), which was reacted with dimethylol propionic acid and isophorone diisocyanate to form a prepolymer. This product was neutralized with triethylamine, and 1,4 butanediol was used as a chain extender, followed by the addition of water. 2aminobenzothiazole (ABT) was incorporated in the WPUDs along with the chain extender antimicrobial agent, and the effect of concentration of ABT antimicrobial activity of coatings was studied. WPUDs with $29 \%$ ABT showed good antimicrobial activity with an approximate $60 \%$ inhibition rate [6].

Smart PU foams called auxetic PU (APU) foams were produced and characterized for antibiotic activities. Antimicrobials like chitosan and Ag were incorporated, and PU foams were tested against Gram-negative and Gram-positive bacteria qualitatively (agar diffusion test) and quantitatively (suspension test) according to EN ISO 20645 and ASTM 2149 standard methods respectively. It was found APU foams containing chitosan and Ag more effective against Staphylococcus aureus than against Escherichia coli. Moreover, the APU foam exhibited the best antibacterial activity against the above-mentioned bacteria [7]. The same authors did a similar study on the correlation between structure and antimicrobial activity after modification of the chemical structure of a narrow spectrum bactericidal peptidomimetic PU as in the previous research. The same fatty acids were used. The modifications are done to convert the narrow PU spectrum active only against gram-negative bacteria such as Escherichia coli, Pseudomonas aeruginosa, and Klebsiella pneumoniae, to a broader spectrum 
activity against gram-positive bacteria Staphylococcus aureus and Staphylococcus epidermidis [8].

A series of facially amphiphilic antimicrobial surfactant like poly(ester urethane) with hydrophobic pendant groups and cationic groups distributed uniformly along the polymer chain exhibited bactericidal activity against gram-negative Escherichia coli and Pseudomonas aeruginosa as well as gram-positive Staphylococcus aureus and Staphylococcus epidermidis. Depending upon the cationic-hydrophobic ratio in the polymer, they cause cytoplasmic membrane disruption [9].

A hydroxyl-terminated quarternary ammonium salt polymer (QAP) added to an open cell PU foam in amounts of 1 , and $5 \mathrm{wt} \%$ was synthesized with isocyanate. For the research Fourier transform infrared analysis (FTIR), Thermogravimetry analysis (TGA), Dynamic mechanic analysis (DMA), and energy-dispersive X-ray fluorescence (EDXRF) were used. Even at a low amount of QAP, the foam showed a very similar structure and thermomechanical properties to the unmodified foam. The results indicated that the addition of small amounts of QAP could significantly improve the biocidal performance of the produced foams [10].

Cobalt incorporated poly (glycerol ester) (Co-PGE) having a 59.3\% degree of branching with up to $5 \%$ w/w Co exhibited antibacterial activity against Escherichia coli, Staphylococcus aureus, Pseudomonas aeruginosa and Candida albicans in a broth microdilution study. PU coatings were prepared by blending of $0.5-32.5 \%$ Co-PGE containing $5 \%$ w/w Co. They demonstrated mild to high active antibacterial effects against Bacillus subtilis, Staphylococcus aureus, Escherichia coli, and Candida albicans in the disk diffusion test. PU with $0.5 \%$ w/w Co-PEG had mild inhibition activity against Staphylococcus aureus, and with $10 \%$ w/w showed a high inhibition activity against Candida albicans. The newly obtained Co-PGE has the potential to be used as an antibacterial agent in polymer coatings for protective surfaces and biomedical devices [11].

Fluorinated WPU containing dimethylol propionamide with guanidine (DMG) group extender shows high bacterial $(99,9 \%)$ efficacy against a broad spectrum of gram-positive and gram-negative bacteria. The increase of DMG in the fluorinated WPU showed excellent thermal properties and high crystallization. These new antibacterial products may provide new insights into the development of protection materials, which could be applied as coatings in various fields in order to prevent microbial contamination [12].

A paper presents a new side-chain quaternizede PU as an antibacterial adhesive; quaternization is done for different time intervals. The degree of quaternization of $\mathrm{N}$-diol units is changed from 13.6 to $99.0 \mathrm{~mol} \%$ and studied its effect on adhesive strength. The increase in the degree of quaternization enhanced PU polarity shifting non-leaching antibacterial behavior to the leaching type but maintaining the high adhesive strength [13].

A research was carried out by synthesis of nine different PUs through variation of quaternary ammonium salt diol (QAS) and isocyanate amounts. The effect of morphological structures on the membrane water vapor transmission rate (WVTR) and antibacterial properties was correlated. In all membranes, the WVTR with the increase of temperature over $10-40^{\circ} \mathrm{C}$ showed water resistance up to a pressure of $2100 \mathrm{~cm}$ water. The WVTR increased by increasing the amount of QAS and decreased by increasing the isocyanate amount. The QAS added membranes provided significant inactivation against Staphylococcus aureus and Escherichia coli [14].

For producing antibacterial catheters, sulfathiazole was introduced in PU. The data from the in vitro drug release study were fit into a mathematical model, and the antibacterial 
efficiency of released sulfathiazole was evaluated by Escherichia coli growth inhibition test [15].

A multifunctional $\mathrm{Ag}_{3} \mathrm{PO}_{4} @ \mathrm{AgBr}-\mathrm{PU} /$ negative ion composite film displays a broadspectrum of antibacterial property against gram-negative Escherichia coli and gram-positive Staphylococcus aureus. The research may provide a simple pathway to construct a highly efficient PU composite film reactor for organic pollutants degradation and bacterial inactivation [16].

\subsection{Drug carriers.}

Micelles pH-responsive and redox sensitive anticancer drug carriers were produced to solve the following two problems: a/. The extracellular stability versus intracellular drug release and b/. The extended bloodstream circulation versus enhanced cell uptake. The micelles were based on PU with pendant carboxyl groups and disulfides connected to diaminohexane. Such micelles being redox-sensitive can rapidly enter tumor cells. They may hold great potential as a bio-triggered drug delivery agent for cancer therapy [17].

Research describes redox-sensitive PU micelles with tunable surface charge switch abilities, cross-linked with $\mathrm{pH}$ cleavable Schiff bonds as anticancer drug carriers. The obtained PU-SS-NH $\mathrm{N}_{2}$ micelles were cross-linked by glutaraldehyde resulting in surface charge suitable and reduction responsive PU micelles. The core cross-linked surface charges switchable PU micelles with the mentioned characteristics may hold great potential for bio-triggered drug delivery for cancer therapy [18].

Unsaturated PU prepolymer was synthesized with 2-hydroxyethyl methacrylate and extended by methyl methacrylate (MMA) to obtain acrylate modified WPU. The study was on the dependence of drug delivery, mechanical, thermal, surface, and structural properties of WPU on the MMA repeating unit content $10 \%-40 \%$. Mitomycin C was used as a model anticancer drug. In vitro and in vivo cytotoxicity evaluation shows that acrylate modified WPUs are biocompatible [19].

Amphiphilic PU based on poly(ethylene glycol) PEG and poly ( $E$-caprolactone)diol with 1-4 arms have been synthesized, and the effects of their number on the properties were studied. By using indomethacin as a model drug, the results showed that the drug loading capacity and in vitro drug sustained release of PU with four arms was the most effective [20].

To solve the problems of poor distribution and delivery of cytotoxic chemotherapeutic drugs, an ultrasound controllable and implantable release-system that uses WPU and chitosan composite membrane as drug carrier with wide flexible loading capacity for DOX is described. The composite films exhibited fined degradability, favorable cytocompatibility, and excellent blood compatibility. In vitro studies showed that the DOX was able to be released slowly in an ultra-sound-controlled manner. Cellular uptake assay and CCK8 assay showed that DOX could be released and taken up by tumor cells [21].

Based on PU microcapsules, a tailor-made traceable $\mathrm{pH}$-sensitive drug delivery PU system using double emulsion technique, containing 3,3'-dioctadecyloxacarbocyanine perchlorate, DOX, and sodium bicarbonate have been produced. Introduced in acid media sodium bicarbonate releases $\mathrm{CO}_{2}$ to puncture the PU-shell leading to the release of DOX to promptly reach the intracellular drug therapeutic threshold to kill the cancer cells in a short time[22].

PU based hydrogel for the controlled release of 5 fluorouracil (5-FU) was prepared using different diisocyanates. In the presence of L-lysine ethyl ester diisocyanate ( LDI), 
wettability, thermal stability, mechanical strength, in vitro biodegradation rate, and protein absorption performance of the hydrogels have higher 5-FU loading and release capacity than the other hydrogels [23].

The research used melt polymerization of a plant oil-based cyclic carbonate monomer with polyether soft segments, and various diamine yielded isocyanate free-segmented poly(amide hydroxyurethane)s (PAHU)s. Electrospinning PAHUs afforded ductile, freestanding fibrous mats suitable for tissue scaffolds applications. PAHU fiber mats exhibited 34 times greater water uptake than the electrospun thermoplastic polyurethane (TPU) control, demonstrating potential application for drug delivery. The findings support the need to continue to study the use of isocyanate-free PU mats in the production of functional biomaterials [24].

\section{PU nanocomposites}

In all medical areas, more additional studies should be done on the production of more advanced components. The presence of fillers, mainly nanofillers in PU matrix, the produced polymer nanocomposites can impart physicomechanical and biological functions (biocompatibility, biodegradability, and bioabsorbability) and still stands for a promising and powerful approach towards the new generation of bioactive materials [25].

\subsection{Antibacterials.}

Many metal nanoparticles like $\mathrm{Ag}, \mathrm{Au}, \mathrm{ZnO}_{2}, \mathrm{TiO}_{2}, \mathrm{NiO}$, and chitosan in $\mathrm{PU}$ matrices can improve their antibacterial activity and enhance thermal stability, mechanical, dynamic mechanical properties, and biostability. The study also established that antibacterial activity of such PU nanocomposites against a wide range of bacteria is enhanced usually at a higher amount of nanoparticles [26].

Uniform distribution of $\mathrm{Ag}$ nanoparticles (nAg) in situ forms on the surface of nanofibers was achieved by adding $\mathrm{AgNO}_{3}$ and tannic acid in a PU solution before electrospinning. Antibacterial activity was tested against Staphylococcus aureus and Escherichia coli bacteria. The PU/nAg nanocomposite showed excellent antibacterial performance [27].

\subsection{Drug carriers.}

Nanocarriers are of paramount significance for drug delivery and nanomedicine technology. Given the imperfect systems and non-ideal therapeutic effects, there is work to be done in synthesis as much as in biological studies, if not more so [28].

A clickable and imageable drug carrier based on multiblock PU (MPUs) having detachable PEG and degradable poly(E-caprolactone) (PCL) as soft segments and the hard ones made of lysine and cysteine derivatives bearing reduction-responsive disulfide linkages and click-active alkynyl moieties was produced. MPUs micelles were prepared by dialysis. The nanovesicles possess attractive core-shell architecture, high loading capacity for DOX and iron oxide nanoparticles, and clickable sites for functionalization. The research provides a promising platform for the development of smart theranostic systems for potential imagingguided cancer treatment and real-time of its detection [29].

By one-pot technique, a new type of glutathione responsive PU based core-shell nanogels with hydrophilic methoxy PEG shell was prepared. MTT and CCK assays indicate that although an obvious lower initial cytotoxicity is compared to free DOX at $24 \mathrm{~h}$ post- 
incubation, the cytotoxicity of the new nanogels loaded with DOX is enhanced after $72 \mathrm{~h}$, which stayed at the similar level with free DOX [30].

PU hydrogels are able to absorb water, biological fluids and, for this reason, are attractive for application in medicine as absorbent or wound healing dressings. The presence of nano organo-montmorillonite (oMMT) in PU improves the swelling capability and slows down the release of active substances. The kinetics swelling and release of paracetamol solution from hydrogel have been studied, and the results confirm the beneficial impact of PU nanocomposite hydrogel on the drug diffusion process [31,32].

Poly(L-histidine)n-S-S-PU-S-S-poly(L-histidine) poly (His)n-S-S-PU-S-S-p(His)n] copolymers having two $\mathrm{pH}$-responsive end blocks and PU middle block tethered by a redoxdisulfide linker have been synthesized. They self-assemble to form micelles, nanodaisies (s) and encapsulate 19\% DOX. The in vitro release profile shows enhanced release of the drug in the acidic medium in the presence of $10 \mathrm{mM}$ glutathione. DOX loaded inhibit the CT26 tumors; $\mathrm{D}$ are considered promising nanocarriers for cancer therapy [33].

An easy way to obtain clay-based multilayers for drug delivery has been produced. Poly (acrylic acid)-laponite (PAA-Lap) nanocomposites were used as a membrane component instead of laponite alone. Coupled with -soluble PU, PU/PAA-Lap multilayer films via layer by layer assembly containing different amounts of Lap were realized. The effect of clay amount on the loading-release behavior of PU/PAA-Lap multilayer was investigated using cationic methylene blue indicator in various $\mathrm{pH}$ solutions. It was found that the presence of Lap can influence the charge density, swelling rate, and $\mathrm{pH}$ stability of the films. With the increasing amount of Lap, the adsorption of indicator solution gradually changed from chemical to physical, while the release mechanism remained as Fickian diffusion. The researchers hope that this method can be used as a model for the production of clay-based polymer drug delivery systems in a low $\mathrm{pH}$ environment [34].

\subsection{Tissue engineering.}

Tissue engineering is a technique applied to replace or repair the body parts to help them regain their primary functions.

The regenerated tissue in PU-graphene 5\% (PU-G5) conduits showed an obvious response in the compound action potential (CAP) examination and had a similar CAP wave pattern to that of the sciatic nerve. The nerve conduit made of PU-G5 had $72 \%$ and $50 \%$ enhancement on the number of blood vessels of regenerated tissue, respectively. The regenerated area of nerve in PU-G5 was 25\% larger than that in pristine PU compared with the U.S. FDA approved conduit Neurotube. The regenerated nerve was 1,7 times more active than that in Neurotube. Besides the fast recovery rate, the ability to regenerate tissue with normal morphology is a significant finding of the research that may lead to clinical applications in the area of mural tissue engineering [35].

Cardiovascular diseases claim an estimated 17.9 million lives globally each year. Nowadays, cardiac tissue engineering has become a promising solution to overcome the drawbacks associated with current therapies. The scaffold used in cardiac tissue engineering must possess thrombo resistant and anticoagulant characteristics to serve as a candidate for cardiovascular applications. In this study, a new PU nanocomposite with carotino oil was produced by electrospinning and was studied with FTIR, contact angle, surface roughness, thermostability, atomic force microscopy (AFM), and TGA. The results showed that developed 
PU/carotino nanocomposite due to better physicochemical and blood compatibility render appropriate potentials for raw materials of cardiac tissue engineering [36].

A novel scaffold made of PU/megni oil nanocomposite was electrospun for tissue engineering applications. The nanofibers were characterized by scanning electron microscopy (SEM), FTIR, TGA, and AFM. The compatibility blood-nanocomposite has been evaluated through activated prothrombin time (APTT), partial thromboplastin time (PT), and hemolysis assay to establish the anticoagulant characteristic. Preliminary investigation with APTT and PT, and hemolysis essay revealed the enhanced anti thrombogenicity nature of the obtained nanocomposite compared to PU. The new nanocomposite membrane might find potential applications as tissue engineering [37].

A developed PU/NiO nanocomposite showed delayed blood clotting time and low hemolytic percentage insinuating the improved anticoagulant characteristic compared to the pristine $\mathrm{PU}$. The patch made with $\mathrm{PU} / \mathrm{NiO}$ nanocomposite rendered better physicochemical, improved blood compatibility, and nontoxicity to the fibroblast cells. Hence the electrospun nanocomposite might serve as a plausible scaffold for cardiac tissue engineering [38].

A newly developed $\mathrm{PU} / \mathrm{TiO}_{2}$ patch exhibiting better physicochemical characteristics enhanced blood compatibility parameters, and proper cell viability rates hold to be a promising candidate also for cardiac tissue engineering [39].

A study investigates the synergistic effect of graphene oxide (GO) nanofibers and polydopamine (PDA) on the osteogenic expression. Contact angle and swelling absorption indicated enhancement and hydrophilicity significantly after coating the scaffolds with PDA, which induced more ability for mineralization bone-like component. According to the obtained data, cell attachment and proliferation were significantly increased in coated constructs, and alkaline phosphatase expression was also increased on the PDA-deposited composite scaffold. The characteristics suggest that the PDA coated PU/GO scaffolds are an appropriate substrate for in vivo studies and the bone regeneration [40].

\section{Other contributions}

Polymer porosity aids in the transfer of fluids through the graft and growth of vascular tissue and allows blood to leak through grafts; therefore, clotting the materials is necessary. In this respect, polymer hydrogels have been synthesized based on acrylic acid and $\mathrm{N}$ hydroxyethyl acrylamide and coated around a porous shape memory polymer (SMP) obtained from lactose functionalized polyurea-urethanes. The research demonstrates the feasibility of hydrogel-coated SMP composite that can maintain the advantages of hydrogel and SMP systems for potential use as vascular grafts [41].

In the case of vascular aneurism, endovascular coils are integrated with PU-urea SMP foams; they have the potential to improve occlusion and reduce coil risks; to enhance their mechanical properties diethanolamine (DEA) was used instead of triethanolamine. The research presents the utility of DEA in SMP synthesis to enable the potential production of safer aneurism treatment [42].

The morphological study of a wound dressing scaffold based on PU/Zn nitrate nanofiber obtained by electrospinning revealed smaller fiber and pore diameters than PU. The research used energy-dispersive X-ray spectroscopy, FTIR, TGA, contact angle measurement, mechanical testing, and AFM. As indicated by the hemolysis and cytocompatibility studies, the presence of $\mathrm{Zn}$ nitrate nanofiber showed a low hemolytic index and enhanced fibroblast proliferation rates. This wound dressing displayed better physicochemical characteristics, 
prolonged blood clotting time, and increased fibroblast proliferation rates, indicating that it might be used as an alternate candidate for wound dressing [43].

Biocompatible poly (ether- block -amide) PEBA copolymers have been widely applied in invasive medical devices. Due to the high hard segment ratios and poor toughness, its modification with TPU is needed to improve this property. When the amount of TPU was $3 \%$ (w/w), the elongation at break and the notched impact strength of PEBA/TPU composite were improved. FTIR revealed that the molecular interaction PEBA-TPU was enhanced due to Hbonding, leading to the tensile strength and toughness increase and low compliance of invasive medical devices [44].

A novel approach deals with a highly porous PU membrane, which has used in tissue engineering [45].

Poly (urethane isocyanate)-type hydrogel synthesized by trimerization of NCOfunctionalized PEG prepolymers is presented as a promising new class of materials for contact lens applications [46].

It was demonstrated that the efficacy of the PU dendrimers through a decrease in free radicals and confirmed their cytoprotective performance over $\mathrm{N}$-acetyl cysteine standard used [47].

The research investigated the effects of radiation in an aqueous environment to determine whether radiation combined with a mimicked in vitro environment is sufficient to change the properties of PU devices. Results from physical, chemical, and mechanical effects confirm that varying dose rates alone do not initiate material changes, which negates the hypothesis that varying dose rates of radiation contribute to complications in peripherally inserted central catheters and central venous catheters [48].

WPU/Fe $\mathrm{F}_{2} \mathrm{O}_{3}$ nanocomposites have been recommended to be used in the field of hydrophobic and microwave absorbent products [49].

Investigations of new polyblend PU/poly (vinyl alcohol) hydrogels showed their possible application as matrices for drug delivery [50].

\section{Remarks}

More additional research has to be done on the finding of more advanced PU products for different medicine areas applications.

Special attention has to be made to the research of new PU nanocomposites applicable in medicine, taking into account that so far many of them having different structures like micelles, hydrogels, and others already proved their promising results.

\section{Funding}

This research received no external funding.

\section{Acknowledgments}

This research has no acknowledgment.

\section{Conflicts of Interest}

The authors declare no conflict of interest. 


\section{References}

1. Kömez, A.; Heper Büyüksungur, S.; Hasirci, V.; Hasirci, N. Effect of chemical structure on properties of polyurethanes: Temperature responsiveness and biocompatibility. Journal of Bioactive and Compatible Polymers 2018, 33.

2. $\quad$ Pivec, T.; Smole, M.; Gašparič, P.; Stana-Kleinschek, K. Polyurethanes for Medical Use. Tekstilec 2017, 60, 182-197, https://doi.org/10.14502/Tekstilec2017.60.182-197.

3. Wilson, C.A.; Chou, S.F.; Lozano, R.; Chen, Y.J.; Neuenschwander, F.P. Thermal and Physico-Mechanical Characterizations of Thromboresistant Polyurethane Films. Bioengineering 2019, 6, https://doi.org/10.3390/bioengineering6030069.

4. Phunphoem, S.; Saravari, O.; Supaphol, P. Synthesis of Cationic Waterborne Polyurethanes from Waste Frying Oil as Antibacterial Film Coatings. International Journal of Polymer Science 2019, 2019, https://doi.org/10.1155/2019/2903158.

5. Kucinska-Lipka, J.; Gubanska, I.; Lewandowska, A.; Terebieniec, A.; Przybytek, A.; Cieśliński, H. Antibacterial polyurethanes, modified with cinnamaldehyde, as potential materials for fabrication of wound dressings. Polymer Bulletin 2019, 76, 2725-2742, https://doi.org/10.1007/s00289-018-2512-x.

6. Mestry, S.U.; Patil, D.M.; Mhaske, S.T. Effect of 2-aminobenzothiazole on antimicrobial activity of waterborne polyurethane dispersions (WPUDs). Polymer Bulletin 2019, 76, 1899-1914, https://doi.org/10.1007/s00289-018-2469-9.

7. Bhullar, S.K.; Orhan, M.; Jun, M.B.G. Antibacterial activity of smart (auxetic) polyurethane foams in Nanotechnology dryven engineered materials Eds. Thomas, S.; Grohens, Y.; Oluwatobi, N.K.; Oluwafemi, S.; Praveen, K.M. Taylor\& Francis 2017; pp. 242-251.

8. Peng, C.; Zhang, T.; Ortiz-Ortiz, D.N.; Vishwakarma, A.; Barton, H.A.; Joy, A. Modification of narrowspectrum peptidomimetic polyurethanes with fatty acid chains confers broad-spectrum antibacterial activity. Polymer International 2019, 68, 1255-1262, https://doi.org/10.1002/pi.5773.

9. Peng, C.; Vishwakarma, A.; Mankoci, S.; Barton, H.A.; Joy, A. Structure-Activity Study of Antibacterial Poly(ester urethane)s with Uniform Distribution of Hydrophobic and Cationic Groups. Biomacromolecules 2019, 20, 1675-1682, https://doi.org/10.1021/acs.biomac.9b00029.

10. Demirci, F.; Yildirim, K.; Kocer, H.B. Antimicrobial open-cell polyurethane foams with quaternary ammonium salts. Journal of Applied Polymer Science 2018, 135, https://doi.org/10.1002/app.45914.

11. Chua, B.W.; Lee, C.S.; Lim, W.H.; Pichika, M.R. One-pot synthesis of cobalt-incorporated polyglycerol ester as an antimicrobial agent for polyurethane coatings. Journal of Applied Polymer Science 2018, 135, https://doi.org/10.1002/app.46045.

12. Zhao, B.; Jia, R.; Zhang, Y.; Liu, D.; Zheng, X. Design and synthesis of antibacterial waterborne fluorinated polyurethane. Journal of Applied Polymer Science 2019, 136, https://doi.org/10.1002/app.46923.

13. Hu, P.; Greiner, A.; Agarwal, S. Synthesis and properties evaluation of quaternized polyurethanes as antibacterial adhesives. Journal of Polymer Science Part A: Polymer Chemistry 2019, 57, 752-757, https://doi.org/10.1002/pola.29321.

14. Aydin, A.; Demirci, F.; Orhan, M.; Kocer, H.B. Preparation of breathable polyurethane membranes with quaternary ammonium salt diols providing durable antibacterial property. Journal of Applied Polymer Science 2019, 136, https://doi.org/10.1002/app.47133.

15. Barde, M.; Davis, M.; Rangari, S.; Mendis, H.C.; De La Fuente, L.; Auad, M.L. Development of antimicrobial-loaded polyurethane films for drug-eluting catheters. Journal of Applied Polymer Science 2018, 135, https://doi.org/10.1002/app.46467.

16. Wang, X.; Jian, J.; Yuan, Z.; Zeng, J.; Zhang, L.; Wang, T.; Zhou, H. In situ loading of polyurethane/negative ion powder composite film with visible light-responsive $\mathrm{Ag}_{3} \mathrm{PO}_{4} @ \mathrm{AgBr}$ particles for photocatalitic and antibacterial applications. Eur.Polym J. 2020, 125.

17. Liu, C.; Guan, Y.; Su, Y.; Zhao, L.; Meng, F.; Yao, Y.; Luo, J. Surface charge switchable and core crosslinked polyurethane micelles as a reduction-triggered drug delivery system for cancer therapy. RSC Advances 2017, 7, 11021-11029, https://doi.org/10.1039/C7RA00346C.

18. Zhao, L.; Liu, C.; Qiao, Z.; Yao, Y.; Luo, J. Reduction responsive and surface charge switchable polyurethane micelles with acid cleavable cross-links for intracellular drug delivery. RSC Advances 2018, 8 , 17888-17897, https://doi.org/10.1039/C8RA01581C.

19. Bahadur, A.; Saeed, A.; Shoaib, M.; Iqbal, S.; Anwer, S. Modulating the burst drug release effect of waterborne polyurethane matrix by modifying with polymethylmethacrylate. Journal of Applied Polymer Science 2019, 136, https://doi.org/10.1002/app.47253.

20. Wang, S.; Zhou, Y.; Zhuang, B.; Chen, H.; Wang, L.; Huang, D.; Zou, T. Synthesis, characterization and effects of arm number on properties of amphiphilic polyurethanes as drug delivery carriers. Journal of Macromolecular Science, Part A 2017, 54, 765-771, https://doi.org/10.1080/10601325.2017.1332467.

21. Feng, Z.; Zheng, Y.; Zhao, L.; Zhang, Z.; Sun, Y.; Qiao, K.; Xie, Y.; Wang, Y.; He, W. An ultrasoundcontrollable release system based on waterborne polyurethane/chitosan membrane for implantable enhanced anticancer therapy. Materials Science and Engineering: C 2019, 104, https://doi.org/10.1016/j.msec.2019.109944. 
22. Niu, Y.; Stadler, F.J.; Song, J.; Chen, S.; Chen, S. Facile fabrication of polyurethane microcapsules carriers for tracing cellular internalization and intracellular $\mathrm{pH}$-triggered drug release. Colloids and Surfaces B: Biointerfaces 2017, 153, 160-167, https://doi.org/10.1016/j.colsurfb.2017.02.018.

23. Kamaci, M. Polyurethane -based hydrogels for controlled drug delivery applications. Eur.Polym.J. 2020, 123.

24. Aduba Jr, D.C.; Zhang, K.; Kanitkar, A.; Sirrine, J.M.; Verbridge, S.S.; Long, T.E. Electrospinning of plant oil-based, non-isocyanate polyurethanes for biomedical applications. Journal of Applied Polymer Science 2018, 135, https://doi.org/10.1002/app.46464.

25. Hu, J.; Tan, L. Chapter 16-Polyurethane composites and nanocomposites for biomedical applications. In: Polyurethane polymer composites and nanocomposites. Thomas, S.; Datta, J.; Hoponiuk, T.J.; Reghunadhan, A. Elsevier 2017; pp. 477-495.

26. Farrokhi, Z.; Ayati, A.; Kanvisi, M.; Sillanpää, M. Recent advance in antibacterial activity of nanoparticles contained polyurethane. Journal of Applied Polymer Science 2019, 136, https://doi.org/10.1002/app.46997.

27. Pant, B.; Park, M.; Park, S.-J. One-Step Synthesis of Silver Nanoparticles Embedded Polyurethane NanoFiber/Net Structured Membrane as an Effective Antibacterial Medium. Polymers 2019, 11, https://doi.org/10.3390/polym11071185.

28. Wang, N.; Cheng, Y.; Li, N.; Wang, H.; Chen, H. The drug loading process of nanocarriers. Adv.Healthc. Mater. 2018, 8 .

29. Wei, J.; Shuai, X.; Wang, R.; He, X.; Li, Y.; Ding, M.; Li, J.; Tan, H.; Fu, Q. Clickable and imageable multiblock polymer micelles with magnetically guided and PEG-switched targeting and release property for $\begin{array}{lllll}\text { precise tumor theranosis. } & \text { Biomaterials }\end{array}$ https://doi.org/10.1016/j.biomaterials.2017.08.005.

30. Qi, D.; Wang, J.; Qi, Y.; Wen, J.; Wei, S.; Liu, D.; Yu, S. One pot preparation of polyurethane-based GSHresponsive core-shell nanogels for controlled drug delivery. Journal of Applied Polymer Science 2020, 137, https://doi.org/10.1002/app.48473.

31. Miotke, M.; Strankowska, J.; Kwela, J.; Strankowski, M.; Józefowicz, M. Transport of paracetamol in swellable and relaxing polyurethane nanocomposite hydrogels. Polymer Bulletin 2020, 77, 483-499, https://doi.org/10.1007/s00289-019-02755-6.

32. Miotke, M.; Strankowska, J.; Kwela, J.; Strankowski, M.; Piszczyk, Ł.; Józefowicz, M.; Gazda, M. Nanosize effect of clay mineral nanoparticles on the drug diffusion processes in polyurethane nanocomposite hydrogels. The European Physical Journal Plus 2017, 132, https://doi.org/10.1140/epjp/i2017-11708-1.

33. John, J.V.; Uthaman, S.; Augustine, R.; Chen, H.; Park, I.K.; Kim, I. pH/redox dual stimuli-responsive sheddable nanodaisies for efficient intracellular tumour-triggered drug delivery. Journal of Materials Chemistry B 2017, 5, 5027-5036, https://doi.org/10.1039/C7TB00030H.

34. Shi, H.; Zhang, R.; Feng, S.; Wang, J. Influence of laponite on the drug loading and release performance of LbL polyurethane/poly(acrylic acid) multilayers. Journal of Applied Polymer Science 2019, 136, https://doi.org/10.1002/app.47348.

35. Lee, T.H.; Yen, C.T.; Hsu, S.H. Preparation of Polyurethane-Graphene Nanocomposite and Evaluation of Neurovascular Regeneration. ACS Biomaterials Science \& Engineering 2020, 6, 597-609, https://doi.org/10.1021/acsbiomaterials.9b01473.

36. Jaganathan, S.K.; Mani, M.P.; Ayyar, M.; Krishnasamy, N.P.; Nageswaran, G. Blood compatibility and physicochemical assessment of novel nanocomposite comprising polyurethane and dietary carotino oil for cardiac tissue engineering applications. Journal of Applied Polymer Science 2018, 135, https://doi.org/10.1002/app.45691.

37. Jaganathan, S.K.; Mani, M.P.; Supriyanto, E. Blood compatibility assessments of electrospun polyurethane nanocomposites blended with megni oil for tissue engineering applications. Anais da Academia Brasileira de Ciências 2019, 91, https://doi.org/10.1590/0001-3765201920190018.

38. Jaganathan, S.K.; Mani, M.P. Enriched mechanical, thermal, and blood compatibility of single stage electrospun polyurethane nickel oxide nanocomposite for cardiac tissue engineering. Polymer Composites 2019, 40, 2381-2390, https://doi.org/10.1002/pc.25098.

39. Jaganathan, S.K.; Mani, M.P.; Sivalingam, S. Augmented physico-chemical, crystalline, mechanical, and biocompatible properties of electrospun polyurethane titanium dioxide composite patch for cardiac tissue engineering. Polymer Composites 2019, 40, 3758-3767, https://doi.org/10.1002/pc.25237.

40. Ghorbani, F.; Zamanian, A.; Aidun, A. Bioinspired polydopamine coating-assisted electrospun polyurethane-graphene oxide nanofibers for bone tissue engineering application. Journal of Applied Polymer Science 2019, 136, https://doi.org/10.1002/app.47656.

41. Dalton, E.; Chai, Q.; Shaw, M.W.; McKenzie, T.J.; Mullins, E.S.; Ayres, N. Hydrogel-coated polyurethane/urea shape memory polymer foams. Journal of Polymer Science Part A: Polymer Chemistry 2019, 57, 1389-1395, https://doi.org/10.1002/pola.29398.

42. Easley, A.D.; Monroe, M.B.B.; Hasan, S.M.; Weems, A.C.; Frederick, J.; Maitland, D.J. Shape memory polyurethane-urea foams with improved toughness. Journal of Applied Polymer Science 2019, 136, https://doi.org/10.1002/app.47268. 
43. Jaganathan, S.K.; Mani, M.P. Single-stage synthesis of electrospun polyurethane scaffold impregnated with zinc nitrate nanofibers for wound healing applications. Journal of Applied Polymer Science 2019, 136, https://doi.org/10.1002/app.46942.

44. Xue, Y.; Tang, Z.; Qin, M.; Yu, M.; Li, Z. Improved toughness of poly(ether-block-amide) via melting blending with thermoplastic polyurethane for biomedical applications. Journal of Applied Polymer Science 2019, 136.

45. Jahid, M.A.; Hu, J.; Thakur, S. Novel approach of making porous polyurethane membrane and its properties for apparel application. Journal of Applied Polymer Science 2020, 137, https://doi.org/10.1002/app.48566.

46. Driest, P.J.; Allijn, I.E.; Dijkstra, D.J.; Stamatialis, D.; Grijpma, D.W. Poly(ethylene glycol)-based poly(urethane isocyanurate) hydrogels for contact lens applications. Polymer International 2020, 69, 131139, https://doi.org/10.1002/pi.5938.

47. Mohamad Ali, B.; Velavan, B.; Sudhandiran, G.; Sridevi, J.; Sultan Nasar, A. Radical dendrimers: Synthesis, anti-tumor activity and enhanced cytoprotective performance of TEMPO free radical functionalized polyurethane dendrimers. European Polymer Journal 2020, https://doi.org/10.1016/j.eurpolymj.2019.109354.

48. Cooke, S.L.; Whittington, A.R. Investigation into Polyurethane at Varying Dose Rates of Ionizing Radiation for Clinical Application. Journal of Chemistry 2018, 2018, https://doi.org/10.1155/2018/7312147.

49. Yang, L.; Fu, Q.; Fu, H. Preparation of novel hydrophobic magnetic Fe3O4/waterborne polyurethane nanocomposites. Journal of Applied Polymer Science 2020, 137, https://doi.org/10.1002/app.48546.

50. Mandur, M.; Bercea, M.; Gradinaru, L.M.; Ciobanu, C.; Drobota, M.; Vlad, S.; Albulescu, R. Investigation of new polyblend PU/poly(vinyl alcohol) hydrogels indicate their possible application as matrices for drug (neomycin sulphate) delivery. Eur.Polym.J. 2019, 118, 137-145. 